\title{
L'entreprise patrimoniale de Louis-Joseph Guyot (1836-1924) à Dourdan, entre érudition et médiation
}

The patrimonial undertaking of Louis-Joseph Guyot (1836-1924) at Dourdan, between erudition and outreach

\section{Léda Martines}

\section{OpenEdition}

Journals

Édition électronique

URL : http://journals.openedition.org/cel/513

DOI : $10.4000 /$ cel. 513

ISSN : 2262-208X

Éditeur

École du Louvre

Édition imprimée

Date de publication : 1 octobre 2013

Référence électronique

Léda Martines, « L'entreprise patrimoniale de Louis-Joseph Guyot (1836-1924) à Dourdan, entre érudition et médiation », Les Cahiers de l'École du Louvre [En ligne], 3 | 2013, mis en ligne le 01 octobre 2013, consulté le 19 avril 2019. URL : http://journals.openedition.org/cel/513; DOI : 10.4000/cel.513

\section{c) (i) (9)}

Les Cahiers de l'École du Louvre sont mis à disposition selon les termes de la licence Creative Commons Attribution - Pas d'Utilisation Commerciale - Pas de Modification 4.0 International. 


\title{
Cahiers de l'École du Louvre recherches en histoire de l'art, histoire des civilisations archéologie, anthropologie et muséologie \\ Numéro 3. Octobre 2013
}

\author{
L'entreprise patrimoniale \\ de Louis-Joseph Guyot (1836-1924) \\ à Dourdan, entre érudition et médiation \\ Léda Martines
}

Article disponible en ligne à l'adresse :

http://www.ecoledulouvre/revue/numero3octobre2013/Martines.pdf

Pour citer cet article :

Léda Martines, « L'entreprise patrimoniale de Louis-Joseph Guyot (1836-1924) à Dourdan, entre érudition et médiation », Cahiers de l'École du Louvre, Recherches en histoire de l'art, histoire des civilisations, archéologie, anthropologie et muséologie [en ligne] no 3, octobre 2013, p. 63 à 72.

Cet article est mis à disposition selon les termes de la Licence Creative Commons Attribution - Pas d'utilisation commerciale - Pas de modification 3.0 non transposé. 


\section{Cahiers de l'École du Louvre recherches en histoire de l'art, histoire des civilisations archéologie, anthropologie et muséologie \\ Numéro 3. Octobre 2013}

\section{Sommaire}

\section{Éditorial}

Équipe de recherche

\section{Dossier: les revues}

Le « GR05 ». Approche de quelques revues du XX $\mathrm{XX}^{\mathrm{e}}$ siècle Introduction d'Hélène Klein

La présentation des objets africains dans DOCUMENTS (1929-1930), magazine illustré

Coline Bidault

Le conflit renaissant de la figure et de l'abstraction dans Labyrinthe, journal mensuel des Lettres et des Arts (octobre 1944-décembre 1946)

Blandine Delhaye

BROOM: An International Magazine of the Arts (1921-1924):

une revue d'avant-garde américaine

Ambre Gauthier

L'image de la guerre dans L'Élan (1915-1916), un refoulement apparent Hadrien Viraben

\section{Articles}

" John Constable et le statut de l'esquisse »

Conférence du 9 avril 2013 dans le cadre du séminaire doctoral

John Murdoch

«Adieu veau, vache, cochon, couvée... » La boucherie à l'Ancien Empire : croisement des données iconographiques, textuelles et archéologiques

Fanny Hamonic

L’entreprise patrimoniale de Louis-Joseph GUYOT (1836-1924)

à Dourdan, entre érudition et médiation

Léda Martines

p. $63-72$

\section{Actualité de la recherche}

Reconnaissance et médiation d'un patrimoine : vecteurs d'une identité territoriale? Journées d'étude de troisième cycle, 14 et 15 juin 2012

Noémie Couillard, Nicolas Navarro, Maylis Nouvellon p. $73-81$

Les « Ymagiers » à l'École du Louvre

Cycle de conférences organisées par l'IRHT 


\section{L'entreprise patrimoniale de Louis-Joseph Guyot (1836-1924) à Dourdan, entre érudition et médiation}

\section{Léda Martines}

La mainmise de l'État sur la sauvegarde des monuments historiques, le développement d'une politique patrimoniale et la professionnalisation croissante des acteurs patrimoniaux au $\mathrm{XX}^{\mathrm{e}}$ siècle, ont contribué à dévaluer la démarche entreprise au siècle précédent. Ces changements ont en effet discrédité l'action de nombreux amateurs qui pourtant ont entrepris au XIX ${ }^{\mathrm{e}}$ siècle de recenser, étudier, faire connaître et valoriser le patrimoine. Louis-Joseph Guyot, avantdernier propriétaire du château de Dourdan se trouve au nombre de ces dilettantes, dont l'œuvre reste ignorée et la mémoire risque même de disparaître.

Depuis que le château de Dourdan est devenu la propriété de la ville en 1964, celle-ci s'emploie à effacer ce qui, dans l'édifice, rappelle le séjour de son ancien occupant. Or ces derniers vestiges constituent la partie émergée de l'entreprise majeure d'un propriétaire peu ordinaire. Dépositaire d'un patrimoine familial dont il mesure l'intérêt public, L.-J. Guyot œuvre dès lors pour la reconnaissance, la sauvegarde et la valorisation du patrimoine local. Cet article entend ainsi réhabiliter l'entreprise de ce personnage afin de défendre l'intérêt de ses interventions au château de Dourdan dont la conservation est aujourd'hui en jeu'

\section{L'érudit passionné}

\section{Un héritier}

Notable fortuné et oisif, Louis-Joseph Guyot présente le profil caractéristique de l'érudit du XIX ${ }^{\mathrm{e}}$ siècle. Il est issu d'un milieu aisé et cultivé, résultat de l'alliance du prestige de la magistrature étampoise et des capitaux du négoce dourdannais ${ }^{2}$. Cet héritage lui permet de bénéficier de la confortable situation de rentier, d'entretenir des liens ancestraux avec Dourdan et de devenir propriétaire - avec sa sœur - de l'ancien château royal de la ville. En 1852, lorsqu'il est mis en vente par la liste civile, cet édifice est acquis par Amédée Guénée, un parent de Louis-Joseph Guyot qui se révèle être un notable dourdannais soucieux de la protection du patrimoine monumental et du passé historique de Dourdan. Onze ans plus tard, A. Guénée lègue le château au père de Louis-Joseph Guyot qui lui-même le transmet à ses enfants ${ }^{3}$. À la mort de sa mère en 1889, L.-J. Guyot rachète à sa sœur la part du château dont elle avait hérité ${ }^{4}$ Il devient alors l'unique propriétaire de l'édifice et en fait dès lors l'objet de ses investigations, de ses lubies et le lieu d'application de ses recherches.

\section{Un châtelain}

Louis-Joseph Guyot a indiscutablement consacré sa vie au château de Dourdan : l'édifice est à la fois son objet d'étude et son œuvre.

\footnotetext{
${ }^{1}$ Le contenu de cet article est issu des recherches que nous avons menées dans le cadre de la rédaction de notre mémoire d'étude, Louis-Joseph Guyot (1836-1924), érudit et acteur du patrimoine de Dourdan, réalisé sous la direction d'Isabelle Bardiès-Fronty conservateur en chef du patrimoine au musée national du Moyen Âge - Thermes et Hôtel de Cluny, et de Jean-Christophe Ton-That, chargé d'études documentaires et responsable du service de la documentation du même musée. Nous les remercions chaleureusement pour leur soutien et leurs conseils avisés. Nos remerciements s'adressent également à Isabelle Mitton-Famié, conservateur du musée du château de Dourdan qui nous a offert la possibilité d'aborder ce sujet et nous a épaulé dans notre travail.

${ }^{2}$ Nous avons été aidés dans nos recherches par les travaux généalogiques de Serge Bouyer, voir : http://gw5.geneanet.org/sergebouyer.

${ }^{3}$ Paris, Archives nationales, Inventaire après décès de Louis-Jacques GUYOT le 14 mai 1864, acte de $M^{e}$ Adolphe de MADRE, Paris, 1864.

${ }^{4}$ Chamarande, Archives départementales de l'Essonne, 48J60, Vente par Madame Juglar à Monsieur Guyot le 19 mars 1889, acte de Me Martin Deslandes notaire à Paris, rue Pierre Marron 66, Paris, 1889.
} 
Ce château médiéval, construit sous l'autorité du roi Philippe-Auguste, présente une disposition caractéristique de l'architecture défensive du début du $\mathrm{XIII}^{e}$ siècle : une enceinte quadrangulaire entourée de fossés maçonnés, et flanquée de neufs tours circulaires en saillie, à chaque angle et au milieu des coursives. Un donjon circulaire isolé de la cour par un large fossé remplace la tour de l'angle nord. Cette structure d'origine a été conservée jusqu'au XIX ${ }^{e}$ siècle mais le monument a connu de multiples remaniements qui en ont passablement modifié l'apparence. Entre le XIII et et le XVIII ${ }^{e}$ siècle plusieurs bâtiments aujourd'hui en partie, voire en totalité disparus, ont occupé successivement le périmètre de sa cour. Au XIX siècle, l'édifice accueille les bureaux de l'Inspection des Eaux et Forêts, la prison et l'école communales. Lorsque Louis-Joseph Guyot en prend possession, le château fort de Dourdan se dresse au milieu de la ville comme une ruine pittoresque que la mousse et les herbes folles ont envahie. "Très amateur du pittoresque et plein de respect pour ce qu'a créé le temps ${ }^{5} »$, l'attention qu'il porte alors au monument participe d'un large phénomène de renaissance de l'art du Moyen Âge. Ainsi L.-J. Guyot est nourri de la vision onirique de l'architecture médiévale véhiculée par les Romantiques, et entreprend d'aménager l'extérieur de l'édifice suivant ces canons. Il laisse la végétation conquérir le bâtiment, recouvrir les façades et dévorer les vestiges de l'ancien château. Il entretient en quelque sorte l'état d'abandon et crée ainsi un décor naturel pour mettre en scène le monument.

FIGURE 1

CHÂTEAU DE DOURDAN FAÇADE DU CHÂTELET ET CLOCHER DE L'ÉGLISE SAINT-GERMAIN DEPUIS LA COUR ENTRE 1890 ET 1924. MU CHATEAU, 2009.0.9.
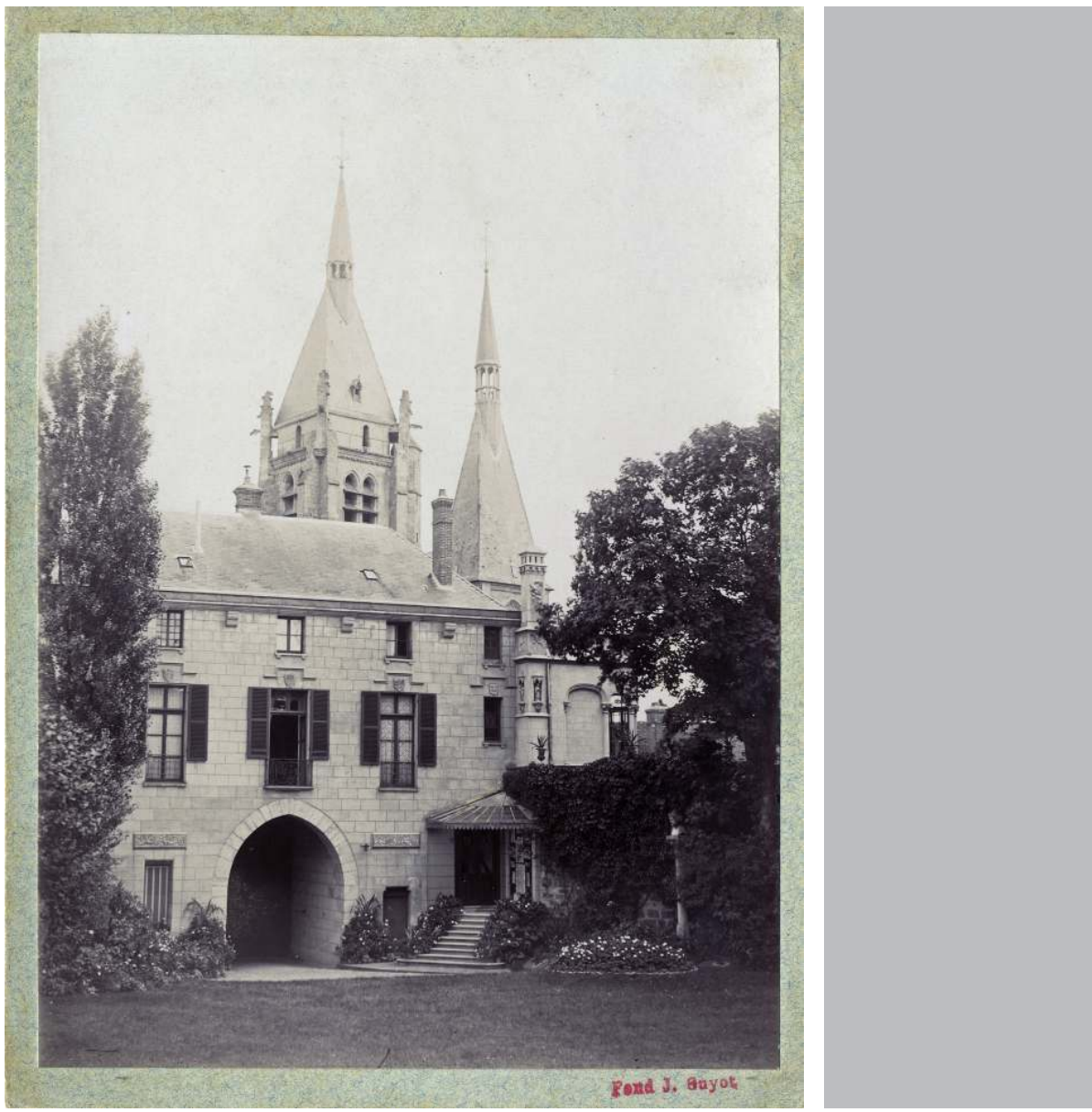

Le corps de logis n'est en revanche pas maintenu dans un état de ruine, L.--J. Guyot le réhabilite en une demeure bourgeoise confortable (fig. 1). Il fait installer plusieurs systèmes de chauffage, cheminées poêles et radiateurs dont les conduits viennent alors hérisser la toiture, modifiant sensiblement l'aspect

\footnotetext{
${ }^{5}$ Louis-Joseph Guyot se décrit en ces termes dans, « L'église et le château de Dourdan, chef-lieu de canton de l'arrondissement de Rambouillet (Seine-et-Oise), lettre à M de Caumont, directeur de la Société française d'archéologie par $M$ Joseph Guyot membre de la Société française d'archéologie », Bulletin Monumental, Caen, 1872, p. 627.
} 
de l'édifice. De même il fait percer des ouvertures dans les tours du châtelet afin d'éclairer plus largement les pièces d'habitation, et dans les combles du grenier à sel qu'il transforme pour installer les logements des domestiques. Il décide également de la construction d'un jardin d'hiver le long de la courtine sud-est et entreprend des aménagements décoratifs. Il réalise ainsi un véritable pastiche : colonnettes, vitraux, arcs ogivaux, quadrilobes et trèfles ornant l'ensemble des pièces de l'habitation, sont autant de formes empruntées au vocabulaire architectural médiéval (fig. 2). Fortement inspiré des travaux de Viollet-le-Duc et de ses élèves, L.-J. Guyot s'emploie même à dessiner des modèles de pièces de mobiliers et d'éléments architecturaux qu'il soumet à des artisans. Il puise également l'inspiration de son décor dans les motifs ornementaux de la Renaissance française et de l'art rocaille du XVIII ${ }^{e}$ siècle. Ces choix décoratifs, déterminés par un recours délibéré à des styles historiques principalement nationaux, sont empreints de l'éclectisme et l'historicisme caractérisant la création artistique du XIX ${ }^{\mathrm{e}}$ siècle, auxquels L.-J. Guyot reste d'ailleurs attaché bien après le tournant du XX ${ }^{\mathrm{e}}$ siècle. Ainsi, loin de mettre en évidence la seule fantaisie du personnage, ces aménagements affirment aussi sa connaissance et son adhésion aux grands courants artistiques qui traversent le $\mathrm{XIX}^{\mathrm{e}}$ siècle.

FIGURE 2

CHÂTEAU DE DOURDAN, VUE DE LA CHAMBRE AU DEUXIĖME ÉTAGE DE LA TOUR SUD DU CHÂTELET, 2009 . (c) FRANÇOIS POCHE

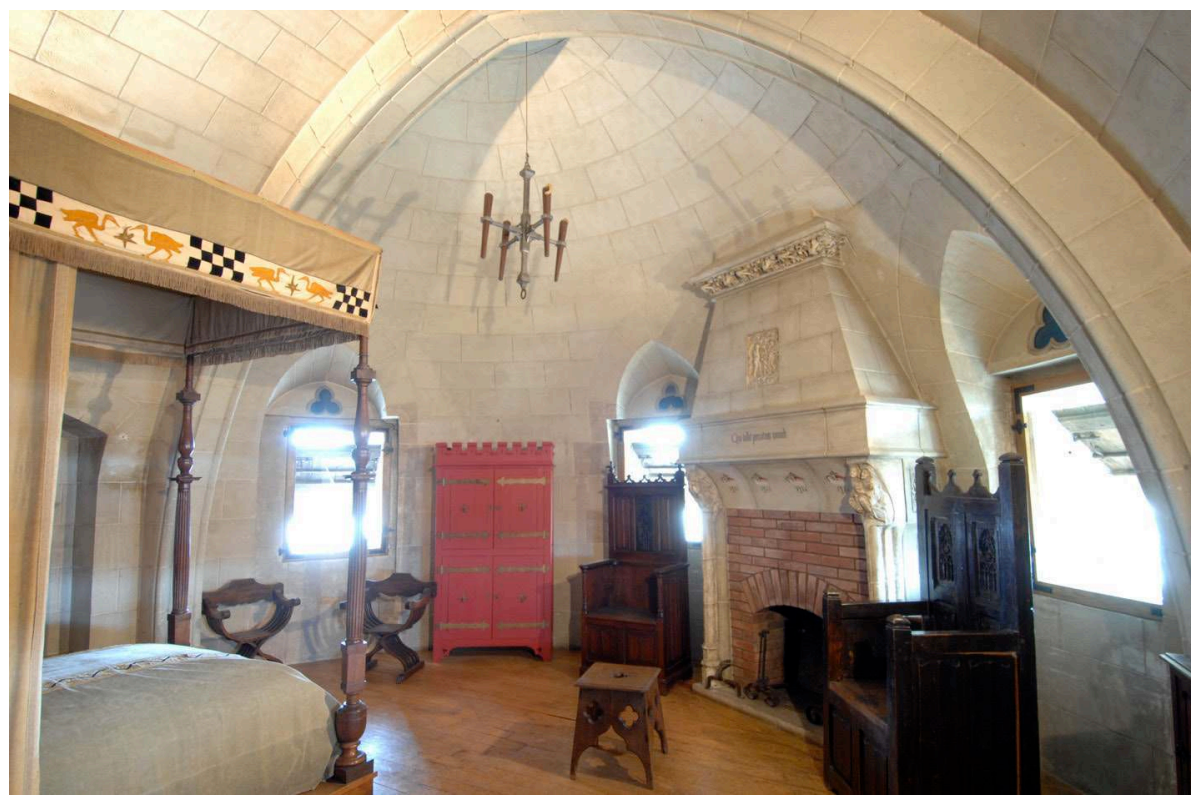

\section{Un chercheur éprouvé}

En 1869, Louis-Joseph Guyot publie son premier ouvrage, la Chronique d'une ville royale: Dourdan, capitale du Hurepoix dans lequel il reconstitue l'histoire de Dourdan, depuis la Préhistoire jusqu'au XIX ${ }^{\mathrm{e}}$ siècle. Conçu comme une entreprise de sauvegarde de la mémoire du passé de la ville, ce volume rassemble en réalité des travaux de différentes natures sous un même titre : études archéologiques, toponymiques, chronique historique, histoire des institutions, et monographies monumentales. Tant par son contenu que par sa forme la Chronique d'une ville royale constitue une œuvre composite symptomatique de l'historiographie locale au XIX ${ }^{\mathrm{e}}$ siècle.

Au regard des données réunies par C.-O. Carbonell sur l'historiographie de la monographie locale ${ }^{6}$, la Chronique de L.-J. Guyot est révélatrice des travaux produits autour des années 1870 . Comme bien des publications historiques locales, cette étude est d'abord héroïque et événementielle ; elle écarte toute analyse idéologique, sociale ou économique. L'histoire locale est en effet principalement étudiée sous le prisme de l'histoire des faits et des personnes car les documents d'archives conservés se rapportent presque exclusivement à l'histoire des institutions. La Chronique d'une ville royale n'en demeure pas moins 
une source de renseignements incontournable pour la connaissance de l'histoire de la ville. L.-J. Guyot, pleinement investi dans la démarche historique de son temps, se distingue par son recours rigoureux et systématique au document d'archive. Sa curiosité s'étend justement à toutes les disciplines qui de près ou de loin enrichissent la connaissance de l'histoire locale et en premier lieu à l'archéologie. Il suit précisément les chantiers de fouilles menées à Dourdan à l'occasion desquels il recueille des artefacts? ${ }^{7}$. Il acquiert également des objets archéologiques et pratique lui-même des fouilles lors des restaurations du donjon du château ${ }^{8}$. L.-J. Guyot s'intéresse également à la sigillographie, l'héraldique et la numismatique, trois sciences dites « auxiliaires de l'histoire » consacrées à l'étude des signes personnels d'autorité et de propriété, et par conséquent indispensables aux recherches historiques, tout particulièrement celles concernant les institutions. Il passe alors commande aux Archives de l'Empire de moulages de sceaux et de médailles pour constituer une collection qui lui sert de référence dans ses analyses?.

Louis-Joseph Guyot est un chercheur rigoureux, animé d'une passion sans limites pour l'histoire locale. Toutefois, lorsqu'il annonce n'avoir « ni d'autres raisons, ni d'autres titres » pour publier sa Chronique que l'amour désintéressé de la connaissance ${ }^{10}$, il a probablement d'autres prétentions. Parmi le lectorat auquel L.-J. Guyot dédie l'ouvrage dans son adresse au lecteur, le premier est celui des érudits. Il inscrit son œuvre au concours des Antiquités de la France organisé par l'Académie des Inscriptions et Belles-Lettres auquel il remporte la première mention honorable. Il prend également le soin d'envoyer une édition de son ouvrage à un certain nombre d'érudits, au nombre desquels se trouve Arcisse de Caumont ${ }^{11}$. La Chronique d'une ville royale constitue en quelque sorte le laisserpassez de Louis-Joseph Guyot pour le milieu savant.

\section{L'acteur du patrimoine}

\section{Un membre actif des sociétés savantes}

Le parcours de Louis-Joseph Guyot au sein du monde érudit révèle une entreprise qui s'inscrit pleinement dans le contexte de prise de conscience patrimoniale développé par le XIX ${ }^{\mathrm{e}}$ siècle. Cette époque voit la création d’un appareil juridique et administratif chargé d'assurer la conservation du « patrimoine » - cet ensemble de productions artistiques que le passé a laissé en héritage - et en particulier du patrimoine monumental. Les institutions patrimoniales nouvellement établies, la Commission des monuments historiques et le Comité des arts et monuments, auxquelles échoient la conservation, l'étude et l'inventaire des monuments, font appel aux connaissances des sociétés savantes. Au XIX ${ }^{e}$ siècle, celles-ci constituent des organismes actifs et honorés, jouant un rôle véritable dans la vie intellectuelle française. Dans ce contexte, Arcisse de Caumont occupe une place essentielle : il met sur pied deux sociétés pionnières destinées à l'étude et à la sauvegarde du patrimoine monumental, la Société des Antiquaires de Normandie en 1824 et la Société française d'archéologie (SFA) en 1834.

Reconnu par ses pairs avec la publication de la Chronique d'une ville royale, Louis-Joseph Guyot devient membre de cette dernière société et intervient en 1872 dans le Bulletin Monumental - organe éditorial de la SFA - avec un article sur le château de Dourdan et l'église Saint-Germain, répondant parfaitement aux préoccupations de la Société. Il présente dans cet article deux brèves monographies monumentales dont la rédaction a été nourrie d'un vocabulaire descriptif et d'une méthode établis par Arcisse de Caumont dans son Abécédaire ou rudiment

\footnotetext{
7 Voir le fragment de céramique issu des fouilles menées en 1868, lors de la construction de la gendarmerie à Dourdan, Musée du château de Dourdan, fonds Guyot, 2006.0.238.

8 Louis-Joseph Guyot, « Lettre de MONSIEUR JOSEPH GUYOT sur les travaux opérés par lui au vieux donjon de Dourdan », dans Mémoires et documents publiés par la Société archéologique de Rambouillet, Rambouillet, 1873, T. I, pp. 253-254.

${ }_{9}$ Dourdan, Musée du château de Dourdan, fonds Guyot, $9 \mathrm{H} 28$, Direction générale des Archives de I'Empire, attestation de délivrance de moulages de sceaux, Paris, 1868.

${ }_{10}$ L.-J. Guyot, Chronique d'une ancienne ville royale Dourdan : capitale du Hurepoix, Paris, 1869, p. 11.

${ }_{11}$ Dourdan, Musée du château de Dourdan, fonds Guyot, 21R20, Arcisse de Caumont, Lettre à M. Guyot, s.I.n.d.
} 
d'archéologie $e^{12}$. En soulignant la qualité de leur architecture et la valeur de leur témoignage historique, L.-J. Guyot met en évidence la capacité des deux édifices dourdannais à faire revivre le passé et à s'adresser à notre sensibilité. Il s'efforce, dans le plaidoyer que constitue cet article, de convertir l'église et le château de Dourdan en monuments historiques ${ }^{13}$. Par ailleurs, sa publication aborde aussi deux questions fondamentales : celles de l'état de conservation des monuments et des interventions de restauration dont ils sont l'objet. L.-J. Guyot s'exprime ainsi à ce propos :

« J'ai beaucoup étudié, beaucoup consulté ; l'alternative n’était pas douteuse : laissé à luimême le monument, dans un avenir plus ou moins prochain, devenait une de ces ruines artistiques précieuses pour le voyageur le témoin vivant du passé pour toute une ville dont il a été l'honneur et la sauvegarde ${ }^{14}$.»

D’achever son article en citant les paroles du secrétaire du Comité des arts et monuments, Adolphe Napoléon Didron :

«En fait de vieux monuments, mieux vaut consolider que réparer, mieux vaut réparer que restaurer, mieux vaut restaurer qu'embellir, et, quoi qu'il arrive, ne jamais supprimer ni démoliir ${ }^{15}$.»

Lorsqu'il aborde ce sujet, L.-J. Guyot semble très au fait des réflexions sur la pratique et la doctrine de la restauration développées par Mérimée, Montalembert et Didron, et dominées par Viollet-le-Duc. L'article, publié dans la revue d'une société aussi éminente que la SFA constitue en conséquence un manifeste : L.-J. Guyot y affirme sa connaissance de l'actualité des débats concernant la sauvegarde des monuments historiques et son engagement pour la cause patrimoniale. Une fois son entreprise légitimée par l'accueil qu'il a reçu de la SFA, il ne participe plus aux publications du Bulletin Monumental mais s'engage auprès de sociétés locales telles que la Société archéologique de Rambouillet (SAR).

Créée en 1836, cette société est une organisation dynamique lorsque Louis-Joseph Guyot y adhère en 1869. Elle fait partie de ces nombreuses sociétés locales d'histoire et d'archéologie dont l'exemple de la SFA a inspiré la fondation. À l'arrivée de L.-J. Guyot, les membres principaux qui la composent sont des érudits également actifs auprès des grandes sociétés parisiennes. Auguste Moutié, le président, membre associé correspondant de la Société des Antiquaires de France pour le département de Seine-et-Oise, et membre non résident du Comité des arts et monuments, s'inscrit directement dans la démarche d'Arcisse de Caumont. Il entend « étudier, décrire et conserver les monuments du passé quels qu'ils soient ${ }^{16}{ }^{\prime}$. Cependant, au même titre que la SFA, et malgré les affirmations de son président Auguste Moutié, la SAR œuvre davantage pour l'étude des monuments anciens que pour leur sauvegarde physique. Les travaux des membres concernent d'abord la publication de documents historiques : cartulaires d'institutions religieuses et nobiliaires de communes. Ils constituent ensuite des monographies monumentales et des biographies de personnages importants pour l'histoire locale. L.-J. Guyot publie un article dans les Mémoires et documents de la Société à propos des travaux qu'il a entrepris sur le donjon du château de Dourdan. Ce sujet, proche de celui abordé dans le Bulletin Monumental, est traité différemment dans la publication de la SAR : L.-J. Guyot insiste moins sur les principes de restauration et le respect de l'authenticité du monument que sur la qualité des vestiges archéologiques mis au jour lors des travaux. Il met alors en évidence l'intérêt de ces découvertes pour l'histoire du château et de Dourdan.

L'engagement de Louis-Joseph Guyot à soutenir l'étude du patrimoine local

12 Louis-Joseph Guyot a du reste fourni à Arcisse de Caumont, le contenu de I'article « château de Dourdan », du chapitre consacré à l'architecture militaire dans son ouvrage Abécédaire ou rudiment d'archéologie, Caen, 1869, pp. 493-495.

${ }^{13}$ L'idée de cette conversion des deux édifices dourdannais s'inspire des réflexions d'Aloïs Riegl sur la notion de monuments historiques dont nous avons pris connaissance par l'intermédiaire de l'ouvrage de Françoise Choay, L'Allégorie du patrimoine, Paris, Seuil, 1996, pp. 124-127.

${ }^{14}$ L.-J. Guyot, op.cit. note 5, pp. 627-628.

15 Id., Ibid., p. 633. Les termes exacts prononcés par Didron lors de la session du Comité historique des arts et monuments de 1839 sont les suivants : «En fait de monuments délabrés, il vaut mieux consolider que réparer, mieux réparer que restaurer, mieux restaurer qu'embellir ; en aucun cas il ne faut supprimer ». Voir le Bulletin archéologique, Paris, 1843, T. 1, p. 47.

${ }^{16}$ Auguste Moutié, «Travaux de M. Moutié, de Rambouillet », Paris, Bulletin Monumental, 1844, pp. 303-305. 
se manifeste aussi par sa participation à la fondation de la Commission des arts et antiquités de Seine-et-Oise (CAASO). Contrairement à la SAR, née d'une initiative locale, la CAASO est une création préfectorale ; elle est initialement fondée pour rédiger et publier les volumes concernant le département de Seineet-Oise, de l'Inventaire général des richesses d'art de la France. Décidée en 1874 par le directeur des Beaux-Arts Philippe de Chennevières, cette entreprise éditoriale vient compléter, dans le domaine des objets mobiliers, le recensement entrepris par les travaux du Comité des arts et monuments dans le domaine monumental. Chennevières compte sur l'érudition locale pour mener l'Inventaire en province, et s'adresse aux présidents des sociétés savantes au même titre qu'aux conservateurs de musées ou aux archivistes pour mener à bien cette mission. La CAASO est ainsi constituée en 1878 pour rassembler les initiatives privées, les canaliser, et servir d'interlocuteur avec l'Administration des Beaux-Arts ${ }^{17}$. Elle est divisée en comités correspondants aux six arrondissements constituants alors le département de Seine-et-Oise ${ }^{18}$; et dès sa mise en place, L.-J. Guyot fait partie des onze membres formant le comité représentant l'arrondissement de Rambouillet. Sa présence à cette place est révélatrice du statut qu'il a dorénavant acquis. Au regard des documents conservés dans le fonds de la CAASO, L.-J. Guyot ne semble avoir participé ni aux réunions trimestrielles de ce comité ni à la rédaction des notices pour élaborer l'Inventaire. Cette attitude étonnante ne réduit pas pour autant la portée de son investissement : peut-être L.-J. Guyot recherche-t-il auprès de cette Commission la légitimation officielle des nombreuses actions qu'il mène à Dourdan en faveur du patrimoine de la ville.

\section{Le médiateur du patrimoine}

Les premiers travaux de Louis-Joseph Guyot sont ainsi prioritairement adressés à l'élite savante dont il souhaite obtenir la considération ; mais une fois sa réputation d'érudit local acquise et même consacrée, L.-J. Guyot entreprend de valoriser plus largement le patrimoine dourdannais.

En 1887, la SAR met en place des excursions, sur le modèle de la SFA, afin de faire connaitre les monuments remarquables de l'arrondissement, et de promouvoir les productions de ses membres. Ces excursions ne sont pas réservées aux seuls adhérents : des élus, des membres d'autres sociétés savantes, des artistes, des femmes - dont l'adhésion aux cercles érudits n'est pas courante - mais aussi des habitants de la région, ou de simples flâneurs y prennent part. L.-J. Guyot a dû accueillir ces manifestations avec enthousiasme car il reçoit les participants de l'excursion à deux reprises, en 1887 et 1892, et Dourdan constitue la première destination de visite de la SAR. Son empressement à conduire ce projet, de même que son dévouement à le concrétiser sont rapportés dans les comptes-rendus de ces journées publiés dans les Mémoires et documents de la Société. Il réserve un accueil chaleureux aux excursionnistes, et propose une visite commentée du château et de l'église, au cours de laquelle il retrace l'histoire des édifices et de la ville. Un texte satirique rédigé par un journaliste venu assister à l'excursion de 1892 relève l'extravagance de ces visites :

«Un lunch est servi (au sommet du donjon), et soit que nous acceptions un Marsala des mains de $\mathrm{M}^{\mathrm{me}}$ Guyot, soit que nous écoutions les descriptions de son mari, nous sommes tous dans l'enchantement d'un accueil si franc et si...pittoresque ${ }^{19}$. »

La passion qui anime L.-J. Guyot et sa volonté de la faire partager à ses hôtes sont néanmoins évidentes. Il laisse la possibilité aux visiteurs d'accéder à la cour et au donjon du château, tous les jours excepté le dimanche, sous la conduite de son concierge ${ }^{20}$. Il établit même un livre d'or afin de recueillir les commentaires

\footnotetext{
17 Concernant les missions de la CAASO voir Commission des arts et antiquités de Seine-et-Oise - Arrêté d'organisation fait à Versailles le 2 septembre 1881.

18 L'ancien département de la Seine-et-Oise était divisé en six arrondissements : Versailles, Étampes, Corbeil, Pontoise, Mantes et Rambouillet. Supprimé en 1968, il correspondait à peu près au regroupement des trois départements actuels que sont I'Essonne, le Val-d'Oise et les Yvelines.

${ }_{19}$ Claude Antoine, «Lettre d'une parisienne sur l'excursion de Dourdan » dans Mémoires de la Société archéologique de Rambouillet, Tours, 1895, T. X, p. 144.

${ }^{20}$ Alexis Martin, Les étapes d'un touriste en France : promenades et excursions dans les environs de Paris, III, Paris, 1896, p. 475.
} 
des visiteurs ${ }^{21}$. Il aménage aussi des meubles-vitrines dans son cabinet de travail (fig. 3) et présente un ensemble lapidaire sous la marquise d'entrée (fig. 4).
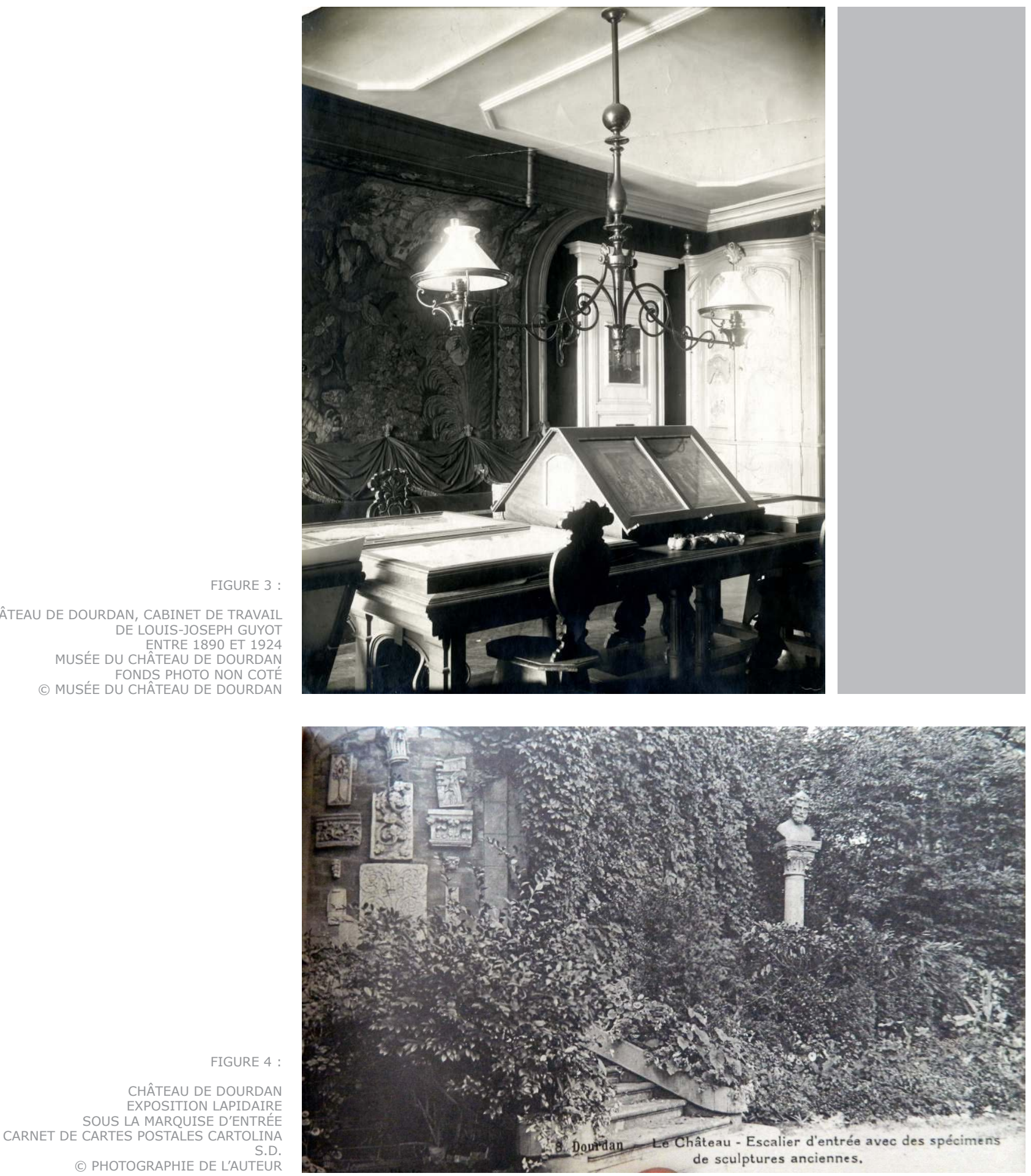

Son exposition se révèle proche du principe du cabinet de curiosités : il rassemble et présente dans sa demeure un ensemble d'objets hétéroclites, dont la plupart entretiennent toutefois un lien avec l'histoire locale, et les utilise comme supports d'étude et de diffusion du savoir. Louis-Joseph Guyot poursuit ainsi son œuvre de valorisation et de vulgarisation en offrant aux participants des excursions, mais aussi à tous ses hôtes, l'exposition d'objets archéologiques, de documents d'archives, de moulages de médailles et de sceaux, qui constituent les sources de sa connaissance. Certes cette exposition ne profite qu'à un nombre 
restreint d'individus, car L.-J. Guyot n'ouvre pas ses appartements aux visites publiques, toutefois elle est pour partie révélée au grand public en 1881. Cette année-là, L.-J. Guyot prête huit objets de sa modeste collection pour l'exposition de "l'art rétrospectif » organisée par la CAASO au château de Versailles, et destinée à valoriser l'intérêt et la qualité du patrimoine mobilier conservé dans le département ${ }^{22}$. L.-J. Guyot propose des objets représentatifs de l'histoire du château et de Dourdan, et sa participation témoigne une nouvelle fois de sa volonté de promouvoir ce patrimoine auprès d'un large public.

Dans cette démarche, Louis-Joseph Guyot cherche tout particulièrement à conquérir les habitants de la commune afin qu'ils s'approprient leur histoire et leurs monuments. À cet effet, il organise plusieurs animations adressées aux Dourdannais. Il compose notamment un drame historique, La prison de La Hire à Dourdan ${ }^{23}$, et programme sa représentation lors de la fête religieuse et patriotique donnée à Dourdan le 17 octobre 1910, pour la béatification de Jeanne d'Arc. La pièce - racontant l'histoire de l'évasion du compagnon d'armes de Jeanne d'Arc emprisonné par les Anglais au château de Dourdan - est jouée dans la salle de la paroisse, en costumes d'époque et devant les Dourdannais, par les jeunes gens du Patronage de la ville (fig. 5). Le concept de la représentation théâtrale, jouée à l'occasion d'une manifestation religieuse n'est pas nouveau en soi : le thêâtre liturgique médiéval a trouvé un écho dans la tradition catholique récente à travers le catéchisme. Pour la composition de sa pièce, L.-J. Guyot s'inspire d'ailleurs largement de ces représentations catéchistiques, mais se nourrit également du théâtre romantique, tel que l'incarne Victor Hugo avec Lucrèce Borgia (1833) ou Marie Tudor (1833), dont il retient les missions : élever l'âme et enseigner l'Histoire. Le propos de La prison de La Hire à Dourdan est donc lié à l'événement programmé par la paroisse, tout en soulignant le rôle de Dourdan et celui du château dans l'histoire de France, valorisant une fois de plus l'intérêt de l'histoire de la ville, et celle de l'édifice.

FIGURE 5

TROUPE DES JEUNES DOURDANNAIS AYANT INTERPRÉTÉ LA PIĖCE DE LOUIS-JOSEPH GUYOT LA PRISON DE LA HIRE Ȧ DOURDAN MUSÉE DU CHÂTEAU DE DOURD MUSÉE DU CHÂTEAU DE DOURDAN FONDS PHOTOGRAPHIQUE NON COTÉ (c) MUSÉE DU CHÂTEAU DE DOURDAN

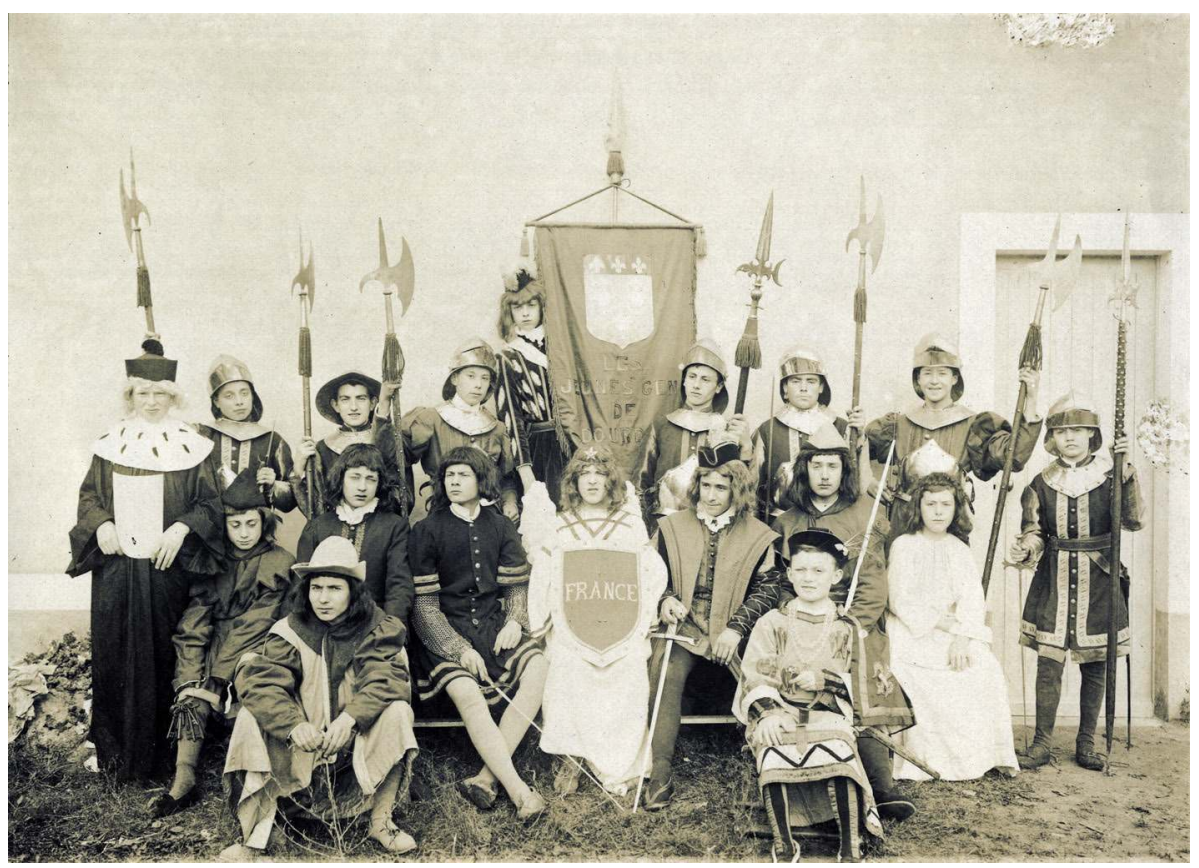

Le texte de cette pièce de théâtre est bien différent de celui rédigé par L.-J. Guyot en 1872 pour le Bulletin Monumental; pourtant les deux écrits défendent l'intérêt du patrimoine dourdannais. Afin de rendre les habitants de Dourdan sensibles à la question patrimoniale, L.-J. Guyot adapte le contenu et la forme de son discours, tente de le rendre plus séduisant. Il mêle ainsi la distraction à l'éducation et utilise le plaisir comme un moyen d'acquérir des connaissances.

22 Exposition de l'art rétrospectif, cat. exp. Palais de Versailles, juin-juillet 1881.

23 Dourdan, Musée du château de Dourdan, fonds Guyot, 3C27, La Prison de La Hire à Dourdan, s.l.n.d. 
Sa démarche est tout à fait intéressante et résolument moderne. La question de savoir pourquoi L.-J. Guyot s'attache à cette entreprise peut se poser. Il semble qu'au-delà de la volonté de faire partager sa passion, Louis-Joseph Guyot soit tout à fait conscient du rôle primordial de chaque habitant dans la protection du patrimoine de la commune. Il relate en effet, dans son article du Bulletin Monumental, comment l'église Saint-Germain de Dourdan est sauvée de la ruine par la mobilisation financière des paroissiens. En sensibilisant les Dourdannais à la question patrimoniale, L.-J. Guyot a donc probablement à l'esprit que « les œuvres d'art doivent avoir autant de gardiens qu'il y a de bons citoyens ${ }^{24} »$.

Largement inspiré des grandes réflexions soulevées par le XIX ${ }^{e}$ siècle sur la conservation des monuments historiques, Louis-Joseph Guyot contribue à enrichir l'énorme masse de connaissances accumulées par les sociétés savantes, mais surtout fait reconnaittre grâce à l'autorité de ces dernières, l'intérêt patrimonial de Dourdan. Tel Arcisse de Caumont envisageant son cours d'archéologie monumentale comme un moyen d'étendre la connaissance et le goût des monuments au-delà du cercle restreint des spécialistes, il s'efforce d'associer la population de Dourdan à son projet. Il met en œuvre des moyens modernes pour sensibiliser les Dourdannais à leur héritage : expositions, visites, conférences et festivités figurent encore aujourd'hui parmi le programme de manifestations proposées par la municipalité. Son rôle dans l'histoire patrimoniale de la ville est manifeste, et soutient encore les pratiques patrimoniales actuelles. Cependant le dédain manifesté par le XX $X^{\mathrm{e}}$ siècle à l'égard de l'œuvre du XIX ${ }^{\mathrm{e}}$ siècle a ouvertement atteint son entreprise et a contribué à l'occulter. À ce titre, ses publications n'ont eu aucune fortune critique et ses interventions architecturales au château de Dourdan ont été discréditées. Après avoir été classé au titre des monuments historiques en 1964, et avoir accueilli un musée au sein de ses murs, l'édifice fait l'objet, entre 1970 et 1990, d'une importante campagne de restauration « afin de mettre le plus possible en valeur la construction médiévale ${ }^{25} »$. Telle est l'idée qui dirige le dégagement des fossés, la restitution de la passerelle d'accès au donjon, la reconstruction des meurtrières, la destruction de la marquise d'entrée ou encore celle du jardin d'hiver. Autant d'interventions qui visent à améliorer la lisibilité de l'édifice médiéval et à mettre en évidence son caractère défensif d'origine, que la transformation en demeure bourgeoise par L.-J. Guyot tendait à faire disparaitre. Ce parti de restauration est motivé par une politique de développement du musée toujours à l'ordre du jour : le renforcement de l'état médiéval du château est conçu comme un faire-valoir privilégié auprès d'un public de visiteurs potentiels. Prétendant rendre au château son apparence d'origine, ces restaurations ont privilégié l'unité de style au détriment de l'authenticité du monument et elles ont surtout sacrifié sous ce prétexte les aménagements de Louis-Joseph Guyot. La réhabilitation de ce personnage contribue de la sorte à faire perdurer son œuvre au château de Dourdan, mais aussi à enrichir la connaissance de l'action de nombreux acteurs privés qui, aux côtés des institutions officielles, ont contribué à l'histoire du patrimoine culturel.

${ }^{24}$ Ces termes sont ceux de I'abbé Grégoire dans son « Rapport sur les destructions opérées par le vandalisme, et sur les moyens de le réprimer » présenté à la Convention le 14 fructidor an II (31 août 1794).

25 Sylvie Ceccaldi, Cabinet Donzet, Quel musée pour Dourdan ? « Programme portant sur la mise en valeur, I'accueil du public, I'animation et l'exploitation du château de Dourdan », s.I., 1985. 


\section{Référence électronique}

Léda Martines, "L'entreprise patrimoniale de Louis-Joseph Guyot (18361924) à Dourdan, entre érudition et médiation », Cahiers de l'École du Louvre. Recherches en histoire de l'art, histoire des civilisations, archéologie, anthropologie et muséologie [en ligne] n 3 , octobre 2013.

URL : http://www.ecoledulouvre.fr/revue/numero3octobre2013/Martines.pdf

\section{(c) creative \\ (B.) $\Theta$}

\section{L'auteur}

Léda Martines a obtenu une licence et un master 1 en double cursus à Paris 1 Panthéon-Sorbonne et à l'École du Louvre où elle a soutenu en 2012 un mémoire d'étude : Lonis-Joseph Guyot (1836-1924), érudit et acteur du patrimoine de Dourdan sous la direction d'Isabelle Bardiès-Fronty et de Jean-Christophe Ton-That. Elle termine actuellement sa $2^{\mathrm{e}}$ année de $2^{\mathrm{e}}$ cycle à l'École du Louvre et travaille à la rédaction d'un mémoire de recherche intitulé : La restauration monumentale selon Henri Révoil (1822-1900), connaissance et interprétation des monuments médiévaux méridionaux. 